\title{
Evolution, Benefits, and Shortcomings of Vaccine Management
}

\author{
Jonathan A. McCullers, MD
}

\begin{abstract}
BACKGROUND: The reduction of childhood mortality by vaccines has been one of the greatest public health successes of the past century. However, many targets for immunization remain uncontrolled, and new or improved vaccines are emerging to meet these challenges.

OBJECTIVE: To review the evolution of vaccination and take an objective look at current vaccine development technologies, thereby framing the discussion of vaccine management.

SUMMARY: The genesis of vaccinology is generally considered to have been a direct result of the observation that persons who had contracted smallpox rarely developed a second case. From this observation, the concept of variolation was born, which involved the inoculation of uninfected individuals using material collected from smallpox lesions with the goal of inducing immunity to future infection. The use of attenuated, live virus to induce immunity was the next step in the evolution of vaccinology, followed by inactivation of the virus when diseases caused by organisms not amenable to attenuation were targeted. More recently, a variety of adjuvant strategies have been developed to improve the immunogenicity of inactivated vaccines, and genetic engineering has been employed to increase the safety, reduce the reactogenicity, and improve the immunogenicity of different vaccines.
\end{abstract}

CONCLUSION: Clinical (efficacy and safety) and economic (cost and profit) considerations are competing priorities that need to be reconciled within a discussion encompassing the government, the public, the pharmaceutical industry, third-party payers, and private individuals or companies who administer these vaccines.

KEYWORDS: Vaccine, History, Management, Adjuvant, Safety

J Manag Care Pharm. 2007;13(7)(suppl S-b):S2-S6

Copyright@ 2007, Academy of Managed Care Pharmacy. All rights reserved.

\section{Author}

JONATHAN A. MCCULLERS, MD, is an associate member, Department of Infectious Diseases, St. Jude Children's Research Hospital, Memphis, Tennessee.

AUTHOR CORRESPONDENCE: Jonathan A. McCullers, MD, Associate Member, Department of Infectious Diseases, St. Jude Children's Research Hospital, 332 N. Lauderdale St., Memphis, TN 38105-2794. Tel: (901) 495-5164; Fax: (901) 495-3099; E-mail: jon.mccullers@ stjude.org
7 he use of vaccination to prevent disease is the greatest public health success of the last century. For many diseases previously considered to be scourges of mankind, including smallpox, polio, diphtheria, and measles, annual mortality has been reduced by more than $99.9 \%$ in the United States ${ }^{1}$ and in many other countries. However, no vaccines are currently licensed for 2 of the 3 most prolific infectious killers, human immunodeficiency virus (HIV) and malaria, and the only vaccine licensed for the third agent, tuberculosis, offers limited effectiveness. In addition, morbidity and mortality remain high for many diseases for which vaccines are used, including influenza, which accounts for 36,000 deaths annually in the United States ${ }^{2}$ and an estimated 500,000 annually worldwide. ${ }^{3}$ Although childhood mortality has been virtually eliminated by the widespread use of pertussis vaccines, morbidity continues to be a problem, accounting for more than 25,000 cases per year in the United States, mostly in adults for whom immunity has waned. ${ }^{4}$ For some newer vaccines, the impact is not yet apparent. An example is a new human papillomavirus (HPV) vaccine (Gardasil), which was licensed in 2006 in the hope of preventing some of the 3,900 annual deaths from cervical cancer related to HPV infection. ${ }^{5}$

\section{The Birth of Vaccinology}

The genesis of vaccinology came through the evolution of attempts to control smallpox. The observation that persons who had contracted smallpox rarely developed a second case suggested the concept of immunity to disease. The Chinese are generally credited with the development of variolation more than a thousand years ago on the basis of this idea, whereby a small amount of dried secretions from a smallpox scab was insufflated into the nose, with the hope of conferring immunity to subsequent exposures. ${ }^{6}$ Modification of this measure in India led to the practice, termed scarification, of scratching the agent into the skin. This method of prevention spread westward into Europe in the 17th century, although results were mixed and significant complications often ensued.

In 1796, Edward Jenner combined the practice of variolation with the observation that milkmaids who had previously contracted cowpox rarely contracted smallpox, and he performed the first immunization by inoculating an 8-year-old boy with cowpox. The boy failed to develop a typical variolation scar upon challenge with smallpox 6 weeks later. ${ }^{7}$ Development of a vaccine based on this principle led to eradication of smallpox in the 20th century.

\section{Attenuation as a Strategy for Vaccine Development}

The discovery of attenuation came serendipitously in the fall of 1881, when Louis Pasteur returned from summer vacation and attempted to inoculate chickens with a culture of Pasteurella 
multocida that he had left out on the laboratory bench. After the chickens failed to show disease, he challenged them with a fresh batch of bacteria and realized that they were protected from the virulent strain because of prior exposure to the attenuated strain. ${ }^{6}$ He coined the term "vaccinate" to describe the use of attenuated organisms to protect against their virulent forms. ${ }^{8}$ After developing chemical methods of attenuation, he went on to develop vaccines against rabies and anthrax. ${ }^{6,9}$ The anthrax vaccine was developed for use in animals and thus served as a forerunner of human anthrax vaccines developed in the 20th century.

Following this lead, French scientists Albert Calmette and Camille Guérin developed the Bacille Calmette-Guérin (BCG) vaccine against tuberculosis by serial passage of Mycobacterium bovis 230 times through artificial media between 1908 and 1921. ${ }^{9}$ BCG was adopted by the League of Nations in 1928, but controversy during a clinical trial in Lubeck in 1929-30 caused Germany to reject the vaccine. A contaminated batch of vaccine caused the deaths during that study of at least 72 infants, leading to a sensationalistic trial of the physicians involved that presaged some of the public-private-governmental tensions that surround vaccine management today. The United States also declined to adopt the vaccine, taking the alternative approach of continuing successful screening programs put into place by the U.S. Public Health Service. ${ }^{9}$

Although the growth of serial cultures in media served to attenuate some bacteria, viruses could not be attenuated by passage in this manner. Some viruses, such as influenza virus or yellow fever virus, could be attenuated by passage in susceptible animals (which led to the development in the 1930s of an attenuated yellow fever vaccine that was used until 1982), ${ }^{10}$ but most viruses did not have an acceptable animal host. The breakthrough came in the 1940s when the team of John Enders, Thomas Weller, and Frederick Robbins developed tissue culture cultivation of viruses and demonstrated that viruses could be attenuated through passage in these cultures. ${ }^{11}$ Their work on the attenuation of poliomyelitis virus won the Nobel Prize in Medicine in 1954.

Numerous viral vaccines have been developed by this method, many of which are still in use today (see Table). One problem that has not been solved by attenuation is the changing antigenicity of influenza viruses that necessitate update of the strains included in the vaccine each year. However, since the genes of influenza are carried on 8 RNA gene segments, a process called reassortment can be used to mix the prevailing strain with a strain attenuated by passage in animals (for the inactivated influenza vaccine) or cell culture (for the live attenuated influenza vaccine) so both antigenicity and attenuation can be achieved. A similar process has been used to develop a rotavirus vaccine that is a reassortant taking antigens from the human virus but attenuating features from a bovine strain. ${ }^{12}$

\section{TABLE Vaccine Development Strategies and Targets}

\section{Inactivated}

\begin{tabular}{l|l} 
Vaccines & Live Vaccines \\
\hline
\end{tabular}

\begin{tabular}{l|l|l|l}
\hline Strategy & Target & Strategy & Target \\
\hline Whole organisms & & $\begin{array}{l}\text { Chemical } \\
\text { attenuation }\end{array}$ & \\
\hline & Typhoid & & Rabies \\
\hline & Cholera & & Anthrax \\
\hline & Plague & & Typhoid \\
\hline & Pertussis & Passage in media & \\
\hline & Influenza & & BCG \\
\hline & Poliovirus & Yellow fever \\
\hline & Hepatitis A & $\begin{array}{l}\text { Passage in } \\
\text { animals }\end{array}$ & \\
\hline
\end{tabular}

\section{Extracts and}

subunits

\begin{tabular}{|c|c|c|c|}
\hline \multicolumn{4}{|l|}{ subunits } \\
\hline & $\begin{array}{l}\text { Japanese } \\
\text { encephalitis }\end{array}$ & & Influenza \\
\hline & Anthrax & $\begin{array}{l}\text { Passage in cell } \\
\text { culture }\end{array}$ & \\
\hline & Influenza & & Poliovirus \\
\hline & Rabies & & Measles \\
\hline \multirow[t]{3}{*}{ Toxoids } & & & Mumps \\
\hline & Diphtheria & & Adenoviruses \\
\hline & Tetanus & & Varicella \\
\hline \multirow[t]{5}{*}{ Polysaccharides } & & & Rotavirus \\
\hline & Pneumococcus & Cold adaptation & \\
\hline & Meningococcus & & Rubella \\
\hline & $\begin{array}{l}\text { H. influenzae } \\
\text { type B }\end{array}$ & & Influenza \\
\hline & Typhoid & & \\
\hline \multicolumn{4}{|l|}{ Conjugation } \\
\hline & $\begin{array}{l}\text { H. influenzae } \\
\text { type B }\end{array}$ & & \\
\hline & Pneumococcal & & \\
\hline & Meningococcal & & \\
\hline \multicolumn{4}{|l|}{$\begin{array}{l}\text { Recombinant } \\
\text { proteins }\end{array}$} \\
\hline & Hepatitis B & & \\
\hline & Pertussis & & \\
\hline & Lyme & & \\
\hline & $\begin{array}{l}\text { Human } \\
\text { papillomavirus }\end{array}$ & & \\
\hline
\end{tabular}

Note: Vaccines for targets in bold type are currently available in the United States. $B C G=$ Bacille Calmette-Guérin. 


\section{Inactivation: A Safer Alternative?}

Although many organisms are amenable to attenuation, not all are, and safety concerns about side effects and the potential for reversion from an attenuated to a virulent form exist. An alternative in many cases has been the use of inactivated organisms. In 1886, Daniel Salmon and Theobald Smith demonstrated the utility of injections of heat-killed organisms to induce immunity in pigeons. ${ }^{8}$ However, whole-cell preparations were quite often toxic, which led to purification of protein components, polysaccharide capsules, or inactivated toxins as preferred immunogens (Table).

A good example is the acellular pertussis vaccine. Using centrifugation techniques, lab technicians separate portions of culture supernatant containing the antigenic proteins from the rest of the material, which contains the inflammatory product lipopolysaccharide (LPS) that is responsible for most of the reactions to whole-cell pertusis vaccines. ${ }^{4}$ However, the reduction in reactogenicity typically comes at a price: reduced immunogenicity. In many cases, high doses of antigen, multiple exposures, or both are needed to induce an effective immune response to inactivated vaccines. In the case of purified polysaccharide vaccines, the polysaccharide itself elicits only B-cellmediated humoral immunity, without the stimulation of T-cells required for immunologic memory and a secondary immune response. ${ }^{13}$ Protection from disease is often short-lived, and some target groups such as infants respond very poorly to the antigens.

\section{Are Adjuvants the Answer?}

The need to improve the immunogenicity of inactivated vaccines has spurred the development of a variety of adjuvant strategies. Adjuvants act by 1 of 2 basic mechanisms: either by improving presentation of the antigens to the immune system or by stimulating the immune system so a more robust or broader response is achieved. This may improve immunogenicity, allow for dose reduction without compromising immunogenicity, or both. Conjugation of inactivated toxoids to purified polysaccharides is one extremely successful form of adjuvantation. This process is responsible for conjugate vaccines directed against Haemophilus influenzae type B, Neisseria meningitidis, and Streptococcus pneumoniae. It is thought to work by improving helper T-lymphocyte responses to the antigen and generating an anamnestic response, particularly in infants who do not respond well to purified polysaccharides without the presence of the toxoid. ${ }^{14}$

The only compounds approved in the United States for concomitant use with vaccines as adjuvants are mineral salts such as calcium phosphate and alum. Since the 1920s, alum as aluminum hydroxide or aluminum phosphate has been the most widely used adjuvant in humans. Its mechanism of action is thought to be creation of a depot site so that slow, sustained release of the antigen improves presentation to the immune system. ${ }^{15}$ Immunostimulatory compounds derived from plants, animals, or bacteria are common components of many adjuvants and act by stimulation of the immune system, usually through activation of Toll-like receptors or other components of innate immunity.

Combination adjuvants are also being tested. An example is the proprietary adjuvant ASO4, a mixture of alum with monophosphoryl lipid A (a detoxified form of LPS), used by GlaxoSmithKline as a component of its investigational cervical cancer vaccine (Cervarix), which is awaiting U.S. Food and Drug Administration (FDA) approval. ${ }^{5}$ The primary antigen is delivered in replication-defective virus-like particles mixed with the adjuvant ASO4. In the molecular biology age, direct expression of cytokines or agonists of the innate immune systems may be used in adjuvantation strategies.

\section{The Era of Genetic Engineering}

Molecular biology and genetic engineering have had a dramatic effect on the field of vaccinology, although many of the important advances have not yet made it into the market. The first success story in this area was the development of the hepatitis B vaccine, which was licensed in $1986 .{ }^{16}$ The surface protein of hepatitis B virus is expressed from a DNA plasmid in yeast cells, purified and adsorbed on alum for injection.

It is likely that all future vaccines will be genetically engineered to increase safety, reduce reactogenicity, and improve immunogenicity. Toward this end, a variety of new technologies have been developed to create, deliver, or enhance vaccines. Important antigens are cloned into DNA plasmids, which can be used directly as vaccines themselves (naked DNA) or used to produce proteins for vaccination as with the hepatitis $B$ vaccine. With some viruses, several proteins can be expressed together and will assemble into pseudo-virions termed virus-like particles (VLPs) that can act as a vaccine or can be used as a delivery vehicle for another antigen. ${ }^{5}$ The powerful technique of creating cloned DNA copies of RNA viruses allows manipulation of the genomes, expression of the involved proteins, or re-creation of entire viruses with custom-made changes. ${ }^{17}$ Manipulation of viral genomes to remove proteins associated with virulence and to allow insertion of foreign genes has resulted in the creation of numerous viral vectors that may be used as vaccine carriers. ${ }^{18}$ In addition to expression of the antigen, these techniques allow for the incorporation of sequences coding other genes of interest, such as cytokines or Toll-like receptor agonists that may act as adjuvants.

These new techniques also offer the promise of preventing or controlling numerous disease states for which effective vaccines have not yet been developed using standard methods. At the top of the priority list are HIV, malaria, and tuberculosis as well as potential agents of bioterrorism. Novel vaccines are in development for a number of common infectious agents for which we have had no success, including respiratory syncytial 
virus (RSV), herpes simplex virus (HSV), and Candida albicans. Amelioration of the symptoms of chronic infections, including HIV and hepatitis B, are being considered, and 1 product directed against the prevention of recurrent, painful outbreaks of varicella zoster is already in the market (the live varicella zoster vaccine, Zostavax).

The strategy of vaccinating pregnant women to protect their newborn babies via transplacental antibody is being explored, as is prevention of a number of noninfectious conditions. For example, treatment of addiction may be possible by vaccinating against nicotine so that it no longer crosses the blood-brain barrier to engage the corresponding receptors. Prevention of pregnancy may be possible by vaccination against chorionic gonadotrophin and luteinizing hormone, key players in preparing the uterus for conception. ${ }^{6}$ Other innovative targets are being considered as these technologies mature.

\section{Challenges in the Management of Vaccines}

Many challenges exist in the management of vaccines currently on the market as well as the many potential vaccines that may enter the market. The most visible issue in the last decade has been high-profile shortages of influenza vaccine and some of the childhood vaccines, particularly new entries into the market. The reasons for this are mainly basic economics. Pharmaceutical companies typically have a low profit margin on vaccines and therefore produce limited supplies. When demand increases, they are often unable to distribute more vaccine to the end user in a timely fashion, and any manufacturing-related delays exacerbate the shortage. This is particularly problematic for vaccines with a defined shelf life, such as influenza, because companies do not want to discard unused vaccine at the end of the season. The result of high development costs, low profit margins, and increasing regulatory hurdles is that few companies are in the marketplace.

The other group that suffers from poor profit margins with vaccines is the front-line caretakers who administer the vaccine. High upfront expenditures are necessary, and profits are low or even negative in some cases. One recent report estimated that the cost to the physician of administering all the currently recommended vaccines to a child from birth to 18 years is $\$ 1,662 .{ }^{19}$ In addition, vaccine administration takes significant time and effort that could be spent on activities with higher profit margins, such as seeing acutely ill patients. ${ }^{20}$

Although vaccines in general often have limited financial benefit for industry and physicians, they have obvious costbenefit advantages for society. Many childhood vaccines save costs to society as a whole, and others provide benefits at a cost considered reasonable for society. ${ }^{21,22}$ As more vaccines come into the marketplace, this dichotomy between cost savings and cost benefit has become more obvious. The shift from targets that cause a great deal of mortality to those that cause significant morbidity and economic loss has led to a careful appraisal of cost-effectiveness compared with the cost of alternate treatments. A comprehensive vaccination policy must take into account the cost of development and production of vaccines, the cost of distribution and administration, the benefits to both individuals and society as a whole, profit for companies and those who administer the vaccines, and the government's role in balancing these various needs.

One noticeable trend in the development and approval of new vaccines has been an emphasis on safety. There is a growing public demand for the safety of vaccines, engendered by a lack of overt disease that creates the perception that vaccines are no longer necessary. This perception is fueled by antivaccination activist groups. As individuals opt out of vaccination programs, herd immunity decreases, reducing the effectiveness of these programs. Herd or community immunity is generally defined as having a large percentage of the population vaccinated in order to prevent the spread of certain infectious diseases. When herd immunity exists, even individuals not vaccinated (such as newborns and those with chronic illnesses) are offered some protection because the disease has little opportunity to spread within the community.

Failure to participate in the "social contract" results in outbreaks of disease and morbidity in unvaccinated populations. ${ }^{23}$ The social contract in this context is the concept that an implicit agreement exists between a people and their government to accept vaccination and its attendant risks for the benefit of not only the individual but also of society as a whole, in return for services and protection of rights provided by the government. The impact on vaccine development of individuals failing to participate for reasons that have no scientific merit is that both real and imagined safety concerns have to be thoroughly addressed in the current climate in order to bring a vaccine to market. New vaccines have to demonstrate a better safety profile than established vaccines, which can create problems balancing immunogenicity and reactogenicity, particularly when adjuvants are considered.

\section{Decision Making in the Era of Multiple Vaccines}

As more vaccines enter the market, decisions on management of those vaccines will become increasingly complex. Two examples serve to illustrate this point.

A new live, attenuated influenza vaccine (LAIV) (FluMist) was licensed for use in 2003 and entered the market at a noncompetitive price level compared with the existing inactivated vaccine. Issues over pricing, reimbursement, and a limited indication have resulted in poor uptake and use of LAIV. However, recent published data indicate that LAIV has superior efficacy in children, ${ }^{24}$ and pricing differences between LAIV and the inactivated vaccine are now minimal. In addition, it is widely anticipated that LAIV will receive an expanded indication from the FDA this year. Physicians, pharmacies, and managed care organizations will now have to make decisions on which vaccine to use or whether 
to offer both, weighing the efficacy data against factors such as cost, availability, and patient choice.

Another example is the projected entry into the market of a second HPV vaccine in the coming year. The FDA-approved HPV vaccine manufactured by Merck (Gardasil) targets HPV serotypes 16 and 18, which cause around 70\% of cervical cancers, as well as 6 and 11, which are the most common causes of noncancerous genital warts, and includes alum as an adjuvant. ${ }^{5}$ A new HPV vaccine produced by GlaxoSmithKline (Cervarix) is expected to enter the market in the upcoming year, targeting only HPV 16 and 18 . However, this vaccine makes use of the novel adjuvant ASO4, which may extend the breadth of protection of the vaccine to cover additional cancer-causing serotypes. If this is substantiated, individuals and organizations may have to weigh the benefit of reducing genital warts against the possibility of preventing additional cases of cervical cancer by choosing between 2 vaccines with similar safety profiles and similar efficacy against their primary targets, HPV 16 and $18 .^{5}$

\section{Conclusions}

New and existing vaccines need to be safe, effective, and costeffective for both society and for those who manage health care programs. In addition, they must be profitable for those who develop, manufacture, and administer the vaccines. It is increasingly obvious that proper stewardship of vaccines requires a partnership between government, industry, health care organizations, and individuals in both academic and private practice. However, the different interests of these parties and competing priorities can create tensions that must be acknowledged and resolved. Navigating the many issues that surround the management of vaccines in today's society is a complex matter, and it is easy to lose sight of the ultimate goal of benefit to public health.

\section{DISCLOSURES}

This article is based on a presentation given by the author at a symposium, "The Value of Preventive Medicine: A Look at Vaccine Management," held April 11, 2007, at the Academy of Managed Care Pharmacy's 19th Annual Meeting and Showcase in San Diego, California. The symposium was supported by an educational grant from GlaxoSmithKline. The author discloses that he has received an honorarium for participation in the presentation and in this supplement. He also discloses that he has been a consultant to and has received honoraria for speaking at seminars for GlaxoSmithKline and MedImmune.

\section{REFERENCES}

1. Centers for Disease Control and Prevention. Achievements in public health, 1900-1999: impact of vaccines universally recommended for children-United States, 1990-1998. MMWR Morb Mortal Wkly Rep. 1999;48:243-48.

2. Thompson WW, Shay DK, Weintraub E, et al. Mortality associated with influenza and respiratory syncytial virus in the United States. JAMA. 2003;289:179-86.

3. Stohr K. Preventing and treating influenza. BMJ. 2003;326:1223-24.

4. Cherry JD. Historical perspective on pertussis and use of vaccines to prevent it. Microbe. 2007;2:139-44.
5. Lowy DR, Schiller JT. Prophylactic human papillomavirus vaccines. J Clin Invest. 2006;116:1167-73.

6. Plotkin SA. Vaccines: past, present and future. Nat Med. 2005;(suppl);11: S5-S11.

7. Baxby D. Edward Jenner's Inquiry; a bicentenary analysis. Vaccine. 1999; 17:301-07.

8. Plotkin SA. Six revolutions in vaccinology. Pediatr Infect Dis J. 2005;24:1-9.

9. Baker JP, Katz SL. Childhood vaccine development: an overview. Pediatr Res. 2004;55:347-56.

10. Barnett ED. Yellow fever: epidemiology and prevention. Clin Infect Dis. 2007;44:850-56.

11. Enders JF, Robbins FC, Weller TH. Classics in infectious diseases. The cultivation of the poliomyelitis viruses in tissue culture by John F. Enders, Frederick C. Robbins, and Thomas H. Weller. Rev Infect Dis. 1980;2:493-504.

12. Dennehy PH. Rotavirus vaccines—an update. Vaccine. 2007;25:3137-41.

13. Stein KE. Thymus-independent and thymus-dependent responses to polysaccharide antigens. J Infect Dis. 1992;165(suppl 1):S49-S52.

14. Bilukha O, Messonnier N, Fischer M. Use of meningococcal vaccines in the United States. Pediatr Infect Dis J. 2007;26:371-76.

15. Aguilar JC, Rodriguez EG. Vaccine adjuvants revisited. Vaccine. 2007; 25:3752-62

16. Stevens CE, Taylor PE, Tong MJ, et al. Yeast-recombinant hepatitis B vaccine. Efficacy with hepatitis B immune globulin in prevention of perinatal hepatitis B virus transmission. JAMA. 1987;257:2612-16.

17. Hoffmann E, Krauss S, Perez D, Webby R, Webster RG. Eight-plasmid system for rapid generation of influenza virus vaccines. Vaccine. 2002;20:3165-70.

18. Liniger M, Zuniga A, Naim HY. Use of viral vectors for the development of vaccines. Expert Rev Vaccines. 2007;6:255-66.

19. Nelson JD, McCracken GHJ. The high cost of vaccines. Pediatr Infect Dis J. 2007;26: yellow pages.

20. Szilagyi PG, Iwane MK, Schaffer S, et al. Potential burden of universal influenza vaccination of young children on visits to primary care practices. Pediatr. 2003;112:821-28.

21. Strutton DR, Stang PE. Prophylaxis against respiratory syncytial virus (RSV), varicella, and pneumococcal infections: economic-based decisionmaking. J Pediatr. 2003;143(suppl):S157-S162.

22. Newall AT, Beutels P, Wood JG, Edmunds WJ, MacIntyre CR. Costeffectiveness analyses of human papillomavirus vaccination. Lancet Infect Dis. 2007;7:289-96

23. Centers for Disease Control and Prevention. Measles-United States, 2005. MMWR Morb Mortal Wkly Rep. 2006;55:1348-51.

24. Belshe RB, Edwards KM, Vesikari T, et al. Live attenuated versus inactivated influenza vaccine in infants and young children. N Engl J Med. 2007;356: 685-96. 